\title{
Metofluthrin: novel pyrethroid insecticide and innovative mosquito control agent*
}

\author{
Kazuya Ujihara, Noritada Matsuo, Tatsuya Mori**, \\ Yoshinori SHONO ${ }^{\dagger}$ and Tomonori IWASAKI ${ }^{\dagger}$ \\ Agricultural Chemicals Research Laboratory, Sumitomo Chemical Co., Ltd., \\ 2-1-4 Takatsukasa, Takarazuka, Hyogo 665-8555, Japan \\ ${ }^{\dagger}$ Environmental Health Division, Sumitomo Chemical Co., Ltd.
}

(Accepted February 20, 2008)

\begin{abstract}
Metofluthrin (SumiOne ${ }^{\circledR}$, Eminence ${ }^{\circledR}$ ) is an exciting novel pyrethroid discovered by Sumitomo Chemical Co., Ltd. It was registered in Japan in January 2005 and is under worldwide development for environmental health use. Metofluthrin has extremely high knockdown activity against various insect pests, especially mosquitoes, as well as high volatility and low mammalian toxicity. This is applicable to not only existing mosquito-controlling devices such as mosquito mats and coils, but also various new formulations and devices such as paper emanators, fan-driven devices, and resin formulations. Metofluthrin is more than 40 times as active as $d$-allethrin against southern house mosquitoes when used in mosquito coils. This paper describes the story behind the discovery of Metofluthrin and its efficacy against mosquitoes in various formulations. (C Pesticide Science Society of Japan
\end{abstract}

Keywords: Metofluthrin, pyrethroid, knockdown, mosquito.

\section{Introduction}

At present, the main devices used for mosquito protection are mosquito coils, electric mosquito mats and liquid vaporizers, but these methods all vaporize insecticides into the air using heating by means of fire or electricity to kill the insects. Most devices contain pyrethroids as active ingredients because of their "knockdown effect," where mosquitoes are rapidly paralyzed and cannot suck blood, and due to their low mammalian toxicity.

Much attention has recently been directed toward the development of non-heated formulations, such as fan vaporizers, because of their increased safety and ease of use, especially during outdoor activities; however, the insecticidal activity and/or the vapor pressures of existing pyrethroids are unsatisfactory to use with such ambient-temperature devices. Therefore, we started our research to find a new pyrethroid with higher vapor action as well as high activity against mosquitoes.

* See part II for the full Japanese article.

** To whom correspondence should be addressed. (C) Pesticide Science Society of Japan

\section{Discovery}

In the 1970s, Ohno and Elliott independently reported norchrysanthemates that showed comparable insecticidal activity to the corresponding chrysanthemates. We directed our attention toward norchrysanthemates because they have a lower molecular weight and showed comparable insecticidal activity to the corresponding chrysanthemates.

We screened various esters, and found that the 2,3,5,6tetrafluorobenzyl ester exhibited little vapor activity at room temperature. We then synthesized the derivatives with substituents at the 4-position on the benzene ring. All analogs had much higher activity against mosquitoes than the compound using the standard topical application method. The relative toxicity peaked with between two and three carbon atoms at the 4-position of the benzene ring, inter alia, the 4methoxymethyl derivative exhibiting the highest lethal potency. We selected methyl, methoxy, and methoxymethyl derivatives, and evaluated their vapor activities in a non-heated formulation. The results clearly demonstrate that a methoxymethyl derivate shows significantly faster action than other compounds in the non-heated formulation. As a consequence of these findings, we chose this methoxymethyl derivate as a new synthetic pyrethroid with high vapor activity against 
mosquitoes.

\section{Physical Properties and Stability}

Metofluthrin is a pale yellow transparent oily liquid. The vapor pressure at $25^{\circ} \mathrm{C}$ is $1.96 \times 10^{-3} \mathrm{~Pa}$, higher than known pyrethroid insecticides. The kinetic viscosity is $19.3 \mathrm{~mm}^{2} / \mathrm{s}$ $\left(20^{\circ} \mathrm{C}\right)$, considered an easily handled level. Metofluthrin is more stable than $d$-allethrin and prallethrin, which are used in heated and unheated vaporization fields, in the sun, and this shows that it is suitable for use in outdoor applications.

\section{Efficacy}

\section{Heated formulation}

Southern house mosquito (Culex quinquefasciatus) is the most important target for mosquito coils throughout global tropical and subtropical zones. Mosquito coils containing $0.005 \%$ Metofluthrin exhibited an efficacy exceeding that of coils containing $0.2 \% d$-allethrin against this species, and its relative efficacy is estimated to exceed 40 times that of $d$-allethrin. To determine the practical effects of Metofluthrin mosquito coils, field tests were conducted using private residences in Bogor, Indonesia. Mosquito coils containing $0.005 \%$ Metofluthrin exhibited an efficacy exceeding that of coils containing $0.3 \% \quad d$-allethrin. The efficacy of Metofluthrin liquid vaporizers was examined against a field strain of Culex quinquefasciatus from Bogor, Indonesia. In this experiment, the relative efficacy ratio was estimated to be over 5 times that of prallethrin.

\section{Non-heated formulation}

The major characteristic of Metofluthrin is vapor activity at room temperature, which is not seen in the majority of knockdown pyrethroids, such as $d$-allethrin and prallethrin. This characteristic enables Metofluthrin to be applied in nonheated formulations such as fan-type, paper and resin emanator. We evaluated the knockdown efficacy of the fan-type formulation containing Metofluthrin against Aedes albopictus in a large-sized chamber $\left(28 \mathrm{~m}^{3}\right)$. In this experiment, Metofluthrin showed high efficacy against Aedes albopictus. Ambient vaporization devices, where the active ingredient is held in paper or resin, and is vaporized without heating or electricity are easy to use, so there are particular expectations for new developments in the field of mosquito control. First, we investigated a device using paper as the carrier. To confirm the efficacy of the paper device, a field experiment was conducted at a home in Malaysia. A Denguri device containing $100 \mathrm{mg}$ of Metofluthrin exhibited strong biting inhibitory effects against Culex quinquefasciatus, exceeding those of coil formulations containing $0.25 \% d$-allethrin. Practical tests using resin formulations were conducted in Vietnam. Resin formulations containing $1 \mathrm{~g}$ of Metofluthrin exhibited excellent spatial repellent effects against Culex quinquefasciatus and Aedes aegypti for at least six weeks, and we confirmed the practicability of this formulation.

\section{Conclusion}

For more than half a century, Sumitomo Chemical has discovered and marketed over 20 pyrethroids with a wide range of chemical and biological characteristics. These pyrethroids have made a significant contribution to improving human health and happiness by controlling insect pests and the diseases and damage they cause in both agricultural and urban environments. We are proud to add Metofluthrin to our product range and are confident it will become a major tool in the battle against mosquitoes and the diseases they transmit. 